UMD-PP-09-048

September, 2009

\title{
Chiral supergravity actions and superforms
}

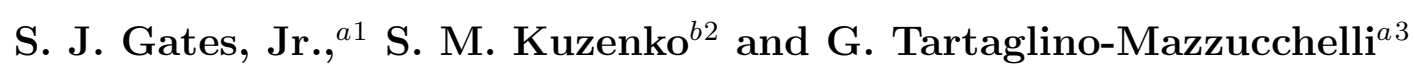 \\ ${ }^{a}$ Center for String and Particle Theory, Department of Physics, University of Maryland \\ College Park, MD 20742-4111, USA \\ ${ }^{b}$ School of Physics M013, The University of Western Australia \\ 35 Stirling Highway, Crawley W.A. 6009, Australia
}

\begin{abstract}
The superform construction of supergravity actions, christened the "ectoplasm method," is based on the use of a closed super $d$-form in the case of $d$ space-time dimensions. In known examples, such superforms are obtained by iteratively solving nontrivial cohomological problems. The latter usually makes this scheme no less laborious than the normal coordinate method for deriving component actions for matter-coupled supergravity. In this note we present an alternative procedure to generate required superforms in four space-time dimensions, which makes use of selfdual vector multiplets. It provides the shortest derivation of chiral actions in two different theories: (i) $\mathcal{N}=1$ old minimal supergravity; and (ii) $\mathcal{N}=2$ conformal supergravity. The $\mathcal{N}=2$ superform construction is developed here for the first time. Although our consideration is restricted to the case of four dimensions, a generalization to higher dimensions is plausible.
\end{abstract}

\footnotetext{
${ }^{1}$ gatess@wam.umd.edu

${ }^{2}$ kuzenko@cyllene.uwa.edu.au

${ }^{3}$ gtm@umd.edu
} 


\section{Introduction}

The power of superspace approaches to supergravity theories in diverse dimensions consists in the possibility to write down the most general locally supersymmetric actions formulated in terms of a few dynamical variables with simple geometric origin. This generality does not come without price to be paid. The point is that, being trivial in principle, a reduction from the parental superfield action to its component counterpart requires some work that is technically quite involved and challenging in many concrete cases.

For off-shell supergravity theories in four dimensions, the component reduction was originally carried out using the Wess-Zumino iterative procedure [1] (see [2, 3] for reviews) and its generalizations [4, 5, 6, 7, 8]. Broadly speaking, this is a technique to reconstruct the relevant density multiplet from its lowest component and the known supersymmetry transformation law, in a suitably chosen Wess-Zumino gauge, order by order in powers of so-called covariant $\Theta$-variables (of mysterious origin). Although this technique can always be applied, at least in principle, in practice it is rather awkward and (unreasonably) laborious. As a result, for some time the issue of component reduction remained the weakest point of superspace formulations for supergravity. 1

This situation has changed with the observation [10] that the concept of superspace normal coordinates [1] can be fruitful for component reduction in supergravity, which has led to the development of more powerful methods [12, 13] (see also [14]). The crucial property of the normal coordinate approaches to component reduction is their universality. They can be used efficiently for any supergravity theory formulated in superspace, and for any number of space-time dimensions. At the same time, these methods are ultimately related to the earlier Noether-like schemes of [1, 2, 5, 6, 7, 8], for the fermionic normal coordinates (which correspond to parallel transport around the bosonic body of curved superspace) can be seen to coincide with the covariant $\Theta$-variables [13].

Over a decade ago, a new universal method 2 for component reduction in supergravity [15, 16] was proposed, sometimes referred to as "ectoplasm," which appears to be more radical than the normal coordinate approach. It presents a superform construction of

\footnotetext{
${ }^{1}$ For supergravity theories possessing prepotential formulations, there exists an alternative, quite systematic scheme for component reduction [9]. Unfortunately, such prepotential formulations are not available in many cases.

${ }^{2}$ The mathematical construction underlying the method of [15, 16] happens to be a special case of the theory of integration over surfaces in supermanifolds developed in [17, 18, 19, see also [20, 21] for related reviews.
} 
supergravity actions, and is based on the use of a closed super $d$-form in the case of $d$ space-time dimensions. Conceptually, it is very simple and its key points can be described in just two paragraphs as follows.

Consider a curved superspace $\mathcal{M}^{d \mid \delta}$ with $d$ space-time and $\delta$ fermionic dimensions, and let $\mathcal{M}^{d \mid \delta}$ be parametrized by local coordinates $z^{M}=\left(x^{\hat{m}}, \theta^{\hat{\mu}}\right)$, where $\hat{m}=1, \ldots, d$ and $\hat{\mu}=1, \ldots, \delta$. The corresponding superspace geometry is described by covariant derivatives

$$
\mathcal{D}_{A}=\left(\mathcal{D}_{\hat{a}}, \mathcal{D}_{\hat{\alpha}}\right)=E_{A}+\Phi_{A}, \quad E_{A}:=E_{A}^{M} \partial_{M}, \quad \Phi_{A}:=\Phi_{A} \cdot \mathbb{J}=E_{A}{ }^{M} \Phi_{M}
$$

Here $\mathbb{I}$ denotes the generators of the structure group (with all indices of $\rrbracket_{S}$ suppressed), $E_{A}$ is the inverse vielbein, and $\Phi=\mathrm{d} z^{M} \Phi_{M}=E^{A} \Phi_{A}$ the connection. As usual, the matrices defining the vielbein $E^{A}:=\mathrm{d} z^{M} E_{M}{ }^{A}$ and its inverse $E_{A}$ are such that $E_{A}{ }^{M} E_{M}{ }^{B}=\delta_{A}{ }^{B}$ and $E_{M}{ }^{A} E_{A}{ }^{N}=\delta_{M}{ }^{N}$. The covariant derivatives obey the algebra

$$
\left[\mathcal{D}_{A}, \mathcal{D}_{B}\right\}=T_{A B}^{C} \mathcal{D}_{C}+R_{A B} \cdot \mathbb{J}
$$

with $T_{A B}^{C}$ the torsion, and $R_{A B}$ the curvature of $\mathcal{M}$.

Next, consider a super $d$-form

$$
J=\frac{1}{d !} \mathrm{d} z^{M_{d}} \wedge \cdots \wedge \mathrm{d} z^{M_{1}} J_{M_{1} \ldots M_{d}}=\frac{1}{d !} E^{A_{d}} \wedge \cdots \wedge E^{A_{1}} J_{A_{1} \ldots A_{d}}
$$

constrained to be closed

$$
\mathrm{d} J=0 \quad \Longleftrightarrow \quad \mathcal{D}_{[B} J_{\left.A_{1} \cdots A_{d}\right\}}-\frac{d}{2} T_{\left[B A_{1} \mid\right.}{ }^{C} J_{\left.C \mid A_{2} \cdots A_{d}\right\}}=0 .
$$

Then, the integral over space-time

$$
S=\frac{1}{d !} \int \mathrm{d}^{d} x \varepsilon^{\hat{m}_{1} \ldots \hat{m}_{d}} J_{\hat{m}_{1} \ldots \hat{m}_{d}}=\frac{1}{d !} \int \mathrm{d}^{d} x \varepsilon^{\hat{m}_{1} \ldots \hat{m}_{d}} E_{\hat{m}_{d}}{ }^{A_{d}} \ldots E_{\hat{m}_{1}}{ }^{A_{1}} J_{A_{1} \ldots A_{d}}
$$

possesses the following fundamental properties: (i) $S$ is independent of the Grassmann variables $\theta$ 's; and (ii) $S$ is invariant under general coordinate transformations on $\mathcal{M}^{d \mid \delta}$ and structure group transformations, and therefore

$$
S=\left.\frac{1}{d !} \int \mathrm{d}^{d} x \varepsilon^{\hat{m}_{1} \ldots \hat{m}_{d}} E_{\hat{m}_{d}}^{A_{d}} \ldots E_{\hat{m}_{1}}^{A_{1}} J_{A_{1} \ldots A_{d}}\right|_{\theta=0} .
$$

In physically interesting cases, the superform $J$ has to obey some additional covariant constraints imposed on its components $J_{A_{1} \ldots A_{d}}$. This is how the dependence of $J$ on the geometric fields in (1.1) and (1.2) occurs. 
As is clear from the above discussion, the ectoplasm method is very general, and its use for component reduction 3 is just one of many possible applications. It is actually a method for constructing supersymmetric invariants. In particular, the method has already been applied to study the structure of higher-order corrections in heterotic string theory [23], as well as for elucidating the structure of higher-loop counterterms in maximally supersymmetric Yang-Mills theories [24]. The last two works are in accord with comments made at the end of the first work in [15], where it was even conjectured that the ectoplasmic concept might find application outside of supersymmetric theories.

Independently of concrete applications, the starting point of the ectoplasm method is always a closed super $d$-form $J$ given explicitly. To construct such a superform, one has to address the cohomology problem (1.4) that is non-trivial in general. For instance, if one somehow fixes a non-vanishing component of $J_{A_{1} \ldots A_{d}}$ of lowest mass dimension and then tries to restore the components of higher dimension by iteratively solving the cohomology equations (1.4), the resulting calculation can be argued to be equivalent to that one encounters when applying the normal coordinate method of [13] (which proves to be more powerful than the scheme presented in [12]). Therefore, in the context of component reduction, one does not gain much if the ectoplasm method is implemented iteratively. However, the present paper is aimed to show that in conjunction with additional ideas this method becomes the most efficient approach to component reduction in supergravity.

Given a $2 n$-dimensional symplectic manifold, its volume $2 n$-form $\Omega$ is known to coincide, modulo a numerical factor, with $\omega^{\wedge n} \equiv \omega \wedge \cdots \wedge \omega$, where $\omega$ is the symplectic two-form, $\mathrm{d} \omega=0$. In this paper we will try to mimic this result in the case of fourdimensional supergravity theories. Specifically, for a given supergravity theory, we will try to engineer the corresponding four-form $J$ from the wedge-product of closed two-forms. It turns out that for this purpose it is sufficient to play with self-dual vectors multiplets (as defined, e.g., in [25] in the flat case) if $\mathcal{N}=1$ and $\mathcal{N}=2$ supergravities are considered.

This paper is organized as follows. In section 2 we illustrate our approach by providing a new simplest/shortest derivation of the closed four-form [16] which corresponds to the chiral action principle within the old minimal formulation for $\mathcal{N}=1$ supergravity. Using the idea described in section 2 , in section 3 we derive a closed four-form that generates the component form of the chiral action principle in $\mathcal{N}=2$ conformal supergravity. The latter result is then recast in the form of a complete density projector formula for a general $\mathcal{N}=2$ locally supersymmetric action. A brief discussion of the results is given in

\footnotetext{
${ }^{3}$ In the context of component reduction, its most recent application has been given in 22 where the density projection formula for $2 \mathrm{D} \mathcal{N}=4$ supergravity was determined.
} 
section 4. The paper is concluded with two technical appendices in which the superspace geometries for $\mathcal{N}=1$ old minimal and $\mathcal{N}=2$ conformal supergravities are reviewed in a concise form.

\section{Chiral action in $\mathrm{N}=1$ old minimal supergravity}

The closed four-form, which corresponds to the chiral action principle within the old minimal formulation for $\mathcal{N}=1$ supergravity, was constructed in [16]. As an illustration of our procedure, in this section we present a new, simplest derivation of this superform. It is based on the use of a self-dual vector multiplet. The latter is described by a complex closed two-form

$$
F=\frac{1}{2} E^{B} \wedge E^{A} F_{A B}, \quad \mathrm{~d} F=0
$$

which is characterized by the following components:

$$
\begin{aligned}
F_{\alpha \beta}=0, & F_{A} \dot{\beta}=0 \\
F_{a \beta}=-\left(\sigma_{a}\right)_{\beta \dot{\beta}} \bar{W}^{\dot{\beta}}, & F_{a b}=-\frac{\mathrm{i}}{2}\left(\tilde{\sigma}_{a b}\right)^{\dot{\alpha} \dot{\beta}} \overline{\mathcal{D}}_{\dot{\alpha}} \bar{W}_{\dot{\beta}}
\end{aligned}
$$

Here the spinor field strength $\bar{W}_{\dot{\alpha}}$ is covariantly antichiral,

$$
\mathcal{D}_{\alpha} \bar{W}_{\dot{\alpha}}=0
$$

and obeys the Bianchi identity

$$
\overline{\mathcal{D}}_{\dot{\alpha}} \bar{W}^{\dot{\alpha}}=0
$$

which implies that the vector multiplet is on-shell. In other words, the explicit expression for the two-form is

$$
F=-E^{\beta} \wedge E^{a}\left(\sigma_{a}\right)_{\beta \dot{\beta}} \bar{W}^{\dot{\beta}}-\frac{\dot{\mathrm{i}}}{4} E^{b} \wedge E^{a}\left(\tilde{\sigma}_{a b}\right)^{\dot{\alpha} \dot{\beta}} \overline{\mathcal{D}}_{\dot{\alpha}} \bar{W}_{\dot{\beta}}
$$

It is an instructive exercise to check explicitly, using the (anti)commutation relations for the covariant derivatives $\mathcal{D}_{A}$ collected in Appendix A, that the complex two-form $F$ defined above is indeed closed, $\mathrm{d} F=0$. Alternatively, the latter property becomes obvious if one recalls the structure of an off-shell real vector multiplet in curved superspace (see [2, 3, 9] for reviews). Its field strength

$$
\mathcal{F}=\mathrm{d} \mathcal{V}=\frac{1}{2} E^{B} \wedge E^{A} \mathcal{F}_{A B}, \quad \mathcal{V}=E^{A} \mathcal{V}_{A}
$$


with $\mathcal{V}$ the gauge field, is characterized by the following components:

$$
\begin{gathered}
\mathcal{F}_{\alpha \beta}=\mathcal{F}_{\alpha \dot{\beta}}=\mathcal{F}_{\dot{\alpha} \dot{\beta}}=0, \\
\mathcal{F}_{\alpha, \beta \dot{\beta}}=2 \varepsilon_{\alpha \beta} \overline{\mathcal{W}}_{\dot{\beta}}, \quad \mathcal{F}_{\dot{\alpha}, \beta \dot{\beta}}=2 \varepsilon_{\dot{\alpha} \dot{\beta}} \mathcal{W}_{\beta}, \\
\mathcal{F}_{\alpha \dot{\alpha}, \beta \dot{\beta}}=\mathrm{i} \varepsilon_{\alpha \beta}\left(\overline{\mathcal{D}}_{\dot{\alpha}} \overline{\mathcal{W}}_{\dot{\beta}}\right)+\mathrm{i} \varepsilon_{\dot{\alpha} \dot{\beta}}\left(\mathcal{D}_{\alpha} \mathcal{W}_{\beta}\right),
\end{gathered}
$$

where the spinor field strength $\mathcal{W}_{\alpha}$ and its conjugate $\overline{\mathcal{W}}_{\dot{\alpha}}$ obey the Bianchi identities

$$
\overline{\mathcal{D}}_{\dot{\alpha}} \mathcal{W}_{\alpha}=\mathcal{D}_{\alpha} \overline{\mathcal{W}}_{\dot{\alpha}}=0, \quad \mathcal{D}^{\alpha} \mathcal{W}_{\alpha}=\overline{\mathcal{D}}_{\dot{\alpha}} \overline{\mathcal{W}}^{\dot{\alpha}}
$$

If the equation of motion for a free vector multiplet is imposed, $\mathcal{D}^{\alpha} \mathcal{W}_{\alpha}=\overline{\mathcal{D}}_{\dot{\alpha}} \overline{\mathcal{W}}^{\dot{\alpha}}=0$, the two sectors of $\mathcal{F}$ which involve the chiral $\mathcal{W}_{\alpha}$ and antichiral $\overline{\mathcal{W}}_{\dot{\alpha}}$ field strengths, respectively, become completely independent, modulo the reality condition. The self-dual vector multiplet is formally obtained by setting $\mathcal{W}_{\alpha}=0$ while keeping the other field strength $\overline{\mathcal{W}}_{\dot{\alpha}}$ non-vanishing.

Consider the closed four-form $J=F \wedge F$,

$$
J=\frac{1}{24} E^{D} \wedge E^{C} \wedge E^{B} \wedge E^{A} J_{A B C D}, \quad \mathrm{~d} J=0
$$

Using eqs. (2.2a $),(2.2 \mathrm{~b})$ and the relations given in Appendix A, one can represent the non-vanishing components of $J$ as follows:

$$
\begin{aligned}
J_{a b \gamma \delta} & =-8 \mathrm{i}\left(\sigma_{a b}\right)_{\gamma \delta} \overline{\mathcal{L}}_{\mathrm{c}} \\
J_{a b c \delta} & =\mathrm{i} \varepsilon_{a b c d}\left(\sigma^{d}\right)_{\delta \dot{\alpha}} \overline{\mathcal{D}}^{\dot{\alpha}} \overline{\mathcal{L}}_{\mathrm{c}} \\
J_{a b c d} & =-\frac{1}{4} \varepsilon_{a b c d}\left(\overline{\mathcal{D}}^{2}-12 R\right) \overline{\mathcal{L}}_{\mathrm{c}} .
\end{aligned}
$$

Here $\overline{\mathcal{L}}_{\mathrm{c}}$ is a covariantly antichiral scalar superfield,

$$
\mathcal{D}_{\alpha} \overline{\mathcal{L}}_{\mathrm{c}}=0
$$

which is expressed in terms of the vector multiplet strength as $\overline{\mathcal{L}}_{\mathrm{c}}=\frac{\mathrm{i}}{2} \bar{W}^{2}$. This representation for $\overline{\mathcal{L}}_{\mathrm{c}}$ is, however, completely irrelevant in order to demonstrate the fact that the four-form $J$ with the non-vanishing components (2.10a - (2.10c) is closed, for eq. (2.11) suffices. At this stage, the self-dual vector multiplet has completed its role and can be forgotten.

Using the closed four-form $J$ associated with an arbitrary covariantly antichiral scalar superfield $\overline{\mathcal{L}}_{\mathrm{c}}$, one can construct a locally supersymmetric action in accordance with the 
general rule (1.6) $)$. It only remains to define the component vierbein $e_{m}{ }^{a}:=\left.E_{m}{ }^{a}\right|_{\theta=0}$ and its inverse $e_{a}{ }^{m}$, such that

$$
e_{a}^{m} e_{m}^{b}=\delta_{a}^{b}, \quad e_{m}^{a} e_{a}^{n}=\delta_{m}^{n}, \quad e:=\operatorname{det}\left(e_{m}{ }^{a}\right)
$$

as well as the gravitino $\Psi_{m}{ }^{\alpha}:=\left.2 E_{m}{ }^{\alpha}\right|_{\theta=0}$ and its tangent-space version $\Psi_{a}^{\alpha}:=e_{a}{ }^{m} \Psi_{m}{ }^{\alpha}$. Then, for the action we obtain

$$
S_{\mathrm{c}}=-\left.\int \mathrm{d}^{4} x e\left(\frac{1}{4} \overline{\mathcal{D}}^{2}-3 R-\frac{\mathrm{i}}{2}\left(\sigma^{d}\right)_{\delta \dot{\alpha}} \Psi_{d}^{\delta} \overline{\mathcal{D}}^{\dot{\alpha}}+\left(\sigma^{a b}\right)_{\gamma \delta} \Psi_{a}^{\gamma} \Psi_{b}{ }^{\delta}\right) \overline{\mathcal{L}}_{\mathrm{c}}\right|_{\theta=0}
$$

This agrees with the results given in [2, 3, 9].

\section{Chiral action in $\mathrm{N}=2$ conformal supergravity}

We now turn to constructing a closed four-form destined to generate the chiral action principle in $\mathcal{N}=2$ conformal supergravity. As shown in [26, 27], $\mathcal{N}=2$ conformal supergravity can be described using the superspace geometry proposed by Grimm [28] which is more economical than the formulation given in [29] (more precisely, the former is obtained from the latter by partially fixing the gauge freedom including the super-Weyl invariance). Appendix B contains all information about the geometric formulation of [28], which is relevant for this paper. A complete presentation can be found in [26].

A self-dual $\mathcal{N}=2$ vector multiplet in curved superspace is described by a complex two-form, $F=\frac{1}{2} E^{B} \wedge E^{A} F_{A B}$, with the following components:

$$
\begin{aligned}
F_{A_{j}}^{\dot{\beta}} & =0, \\
F_{\alpha \beta}^{i j} & =-2 \varepsilon_{\alpha \beta} \varepsilon^{i j} \bar{W}, \quad F_{a \beta}^{j}=\frac{\mathrm{i}}{2}\left(\sigma_{a}\right)_{\beta}{ }^{\dot{\gamma}} \overline{\mathcal{D}}_{\dot{\gamma}}^{j} \bar{W}, \\
F_{a b} & =-\frac{1}{8}\left(\left(\tilde{\sigma}_{a b}\right)_{\dot{\alpha} \dot{\beta}}\left[\overline{\mathcal{D}}^{\dot{\alpha} k} \overline{\mathcal{D}}_{k}^{\dot{\beta}}-4 \bar{Y}^{\dot{\alpha} \dot{\beta}}\right]+4\left(\sigma_{a b}\right)_{\alpha \beta} W^{\alpha \beta}\right) \bar{W} .
\end{aligned}
$$

Thus the field strength $F_{A B}$ is generated by a single scalar superfield $\bar{W}$ which is covariantly antichiral,

$$
\mathcal{D}_{\alpha}^{i} \bar{W}=0
$$

and subject to the equation of motion

$$
\left(\overline{\mathcal{D}}_{\dot{\gamma}}^{(i} \overline{\mathcal{D}}^{\dot{\gamma} j)}+4 \bar{S}^{i j}\right) \bar{W}=0 .
$$


Here the tensor superfields $W^{\alpha \beta}, \bar{Y}^{\dot{\alpha} \dot{\beta}}$ and $\bar{S}^{i j}$ are components of the superspace torsion, see Appendix B. It follows from (3.1C) that $F_{a b}$ possesses both self-dual $\left(F_{\dot{\alpha} \dot{\beta}}\right)$ and antiself-dual $\left(F_{\alpha \beta}\right)$ components.

$$
F_{\dot{\alpha} \dot{\beta}}=\frac{1}{8}\left[\overline{\mathcal{D}}_{\dot{\alpha}}^{k} \overline{\mathcal{D}}_{\dot{\beta} k}-4 \bar{Y}_{\dot{\alpha} \dot{\beta}}\right] \bar{W}, \quad F_{\alpha \beta}=-\frac{1}{2} W_{\alpha \beta} \bar{W} .
$$

However, the latter originates solely due to the curved superspace geometry. Indeed, $F_{\alpha \beta}$ is proportional to the super-Weyl tensor $W_{\alpha \beta}$, and hence it vanishes in the flat superspace limit.

The above relations imply that the two-form $F$ is closed.

$$
\mathrm{d} F=0
$$

To justify this claim, it is sufficient to consider an off-shell real $\mathcal{N}=2$ vector multiplet in curved superspace, $\mathcal{F}=\frac{1}{2} E^{B} \wedge E^{A} \mathcal{F}_{A B}$, which is described in detail in [26]. Its components are expressed in terms of a covariantly chiral superfield $\mathcal{W}, \overline{\mathcal{D}}_{\dot{\alpha}}^{i} \mathcal{W}=0$, and its conjugate $\overline{\mathcal{W}}$ which are related to each other by the Bianchi identity 4

$$
\left(\mathcal{D}^{\gamma(i} \mathcal{D}_{\gamma}^{j)}+4 S^{i j}\right) \mathcal{W}=\left(\overline{\mathcal{D}}_{\dot{\gamma}}^{(i} \overline{\mathcal{D}}^{j) \dot{\gamma}}+4 \bar{S}^{i j}\right) \overline{\mathcal{W}} .
$$

On the mass shell, when the expressions in both sides of (3.6) vanish, one can consistently switch off $\mathcal{W}$ while keeping $\overline{\mathcal{W}}$ non-vanishing. This results in the self-dual $\mathcal{N}=2$ vector multiplet introduced.

Now, consider the closed four-form $J=F \wedge F$,

$$
J=\frac{1}{24} E^{D} \wedge E^{C} \wedge E^{B} \wedge E^{A} J_{A B C D}, \quad \mathrm{~d} J=0 .
$$

It is an edifying calculation to verify that the non-vanishing components of $J$ can be represented as follows:

$$
\begin{aligned}
J_{\alpha \beta \gamma \delta}^{i j k l}= & -32 \mathrm{i}\left(\varepsilon_{\alpha \beta} \varepsilon_{\gamma \delta} \varepsilon^{i j} \varepsilon^{k l}+\varepsilon_{\alpha \gamma} \varepsilon_{\delta \beta} \varepsilon^{i k} \varepsilon^{l j}+\varepsilon_{\alpha \delta} \varepsilon_{\beta \gamma} \varepsilon^{i l} \varepsilon^{j k}\right) \overline{\mathcal{L}}_{\mathrm{c}}, \\
J_{a \beta \gamma \delta}^{j k l}= & -4\left(\varepsilon_{\gamma \delta} \varepsilon^{k l}\left(\sigma_{a}\right)_{\beta}{ }^{\dot{\alpha}} \overline{\mathcal{D}}_{\dot{\alpha}}^{j}+\varepsilon_{\delta \beta} \varepsilon^{l j}\left(\sigma_{a}\right)_{\gamma}{ }^{\dot{\alpha}} \overline{\mathcal{D}}_{\dot{\alpha}}^{k}+\varepsilon_{\beta \gamma} \varepsilon^{j k}\left(\sigma_{a}\right)_{\delta}{ }^{\dot{\alpha}} \overline{\mathcal{D}}_{\dot{\alpha}}^{l}\right) \overline{\mathcal{L}}_{\mathrm{c}}, \\
J_{a b}{ }_{\gamma \delta \delta}= & \mathrm{i}\left(\varepsilon_{\gamma \delta} \varepsilon^{k l}\left(\tilde{\sigma}_{a b}\right)_{\dot{\beta} \dot{\gamma}} \overline{\mathcal{D}}^{\dot{\beta} \dot{\gamma}}+2\left(\sigma_{a b}\right)_{\gamma \delta} \overline{\mathcal{D}}^{k l}+16\left(\sigma_{a b}\right)_{\gamma \delta} \bar{S}^{k l}\right. \\
& \left.-8 \varepsilon_{\gamma \delta} \varepsilon^{k l}\left(\left(\sigma_{a b}\right)_{\alpha \beta} W^{\alpha \beta}-\left(\tilde{\sigma}_{a b}\right)_{\dot{\alpha} \dot{\beta}} \bar{Y}^{\dot{\alpha} \dot{\beta}}\right)\right) \overline{\mathcal{L}}_{\mathrm{c}},
\end{aligned}
$$

\footnotetext{
${ }^{4} \mathrm{Eq}$. (3.6) is a curved-superspace extension of the Bianchi identity given in [30].
} 


$$
\begin{aligned}
J_{a b c \delta}^{l}= & -\mathrm{i} \varepsilon_{a b c d}\left(\frac{1}{6}\left(\sigma^{d}\right)_{\delta \dot{\alpha}} \overline{\mathcal{D}}_{q}^{\dot{\alpha}} \overline{\mathcal{D}}^{l q}+\frac{5}{3}\left(\sigma^{d}\right)_{\delta \dot{\alpha}} \bar{S}^{l q} \overline{\mathcal{D}}_{q}^{\dot{\alpha}}-\left(\tilde{\sigma}^{d}\right)^{\dot{\beta} \alpha} W_{\alpha \delta} \overline{\mathcal{D}}_{\dot{\beta}}^{l}\right. \\
& \left.-\left(\sigma^{d}\right)_{\delta \dot{\alpha}} \bar{Y}^{\dot{\alpha} \dot{\beta}} \overline{\mathcal{D}}_{\dot{\beta}}^{l}+\frac{4}{3}\left(\sigma^{d}\right)_{\delta \dot{\alpha}}\left(\overline{\mathcal{D}}_{q}^{\dot{\alpha}} \bar{S}^{l q}\right)\right) \overline{\mathcal{L}}_{\mathrm{c}} \\
J_{a b c d}= & \varepsilon_{a b c d}\left(\frac{1}{96}\left(\overline{\mathcal{D}}^{i j} \overline{\mathcal{D}}_{i j}-\overline{\mathcal{D}}^{\dot{\alpha} \dot{\beta}} \overline{\mathcal{D}}_{\dot{\alpha} \dot{\beta}}\right)+\frac{2}{3} \bar{S}^{i j} \overline{\mathcal{D}}_{i j}-\frac{1}{3} \bar{Y}^{\dot{\gamma} \dot{\delta}} \overline{\mathcal{D}}_{\dot{\gamma} \dot{\delta}}+\frac{2}{3}\left(\overline{\mathcal{D}}_{\dot{\beta}}^{i} \bar{S}_{i j}\right) \overline{\mathcal{D}}^{\dot{\beta} j}\right. \\
& \left.+\frac{1}{6}\left(\overline{\mathcal{D}}^{i j} \bar{S}_{i j}\right)+3 \bar{S}^{i j} \bar{S}_{i j}-\left(\bar{Y}^{\dot{\alpha} \dot{\beta}} \bar{Y}_{\dot{\alpha} \dot{\beta}}-W^{\alpha \beta} W_{\alpha \beta}\right)\right) \overline{\mathcal{L}}_{\mathrm{c}}
\end{aligned}
$$

Here the scalar $\overline{\mathcal{L}}_{\mathrm{c}}$ is covariantly antichiral,

$$
\mathcal{D}_{\alpha}^{i} \overline{\mathcal{L}}_{\mathrm{c}}=0
$$

and is related to the vector multiplet strength as $\overline{\mathcal{L}}_{\mathrm{c}}=\frac{\mathrm{i}}{4} \bar{W}^{2}$. The operators $\overline{\mathcal{D}}_{i j}$ and $\overline{\mathcal{D}}_{\dot{\alpha} \dot{\beta}}$ in (3.8e) are defined as

$$
\overline{\mathcal{D}}_{i j}:=\overline{\mathcal{D}}_{\dot{\gamma}(i} \overline{\mathcal{D}}_{j)}^{\dot{\gamma}}, \quad \overline{\mathcal{D}}^{\dot{\alpha} \dot{\beta}}:=\overline{\mathcal{D}}_{k}^{(\dot{\alpha}} \overline{\mathcal{D}}^{\dot{\beta}) k}
$$

They possess the following useful identities:

$$
\begin{aligned}
\overline{\mathcal{D}}_{i}^{\dot{\alpha}} \overline{\mathcal{D}}^{\dot{\beta} \dot{\gamma}} U & =\frac{2}{3}\left(\varepsilon^{\dot{\alpha}(\dot{\beta}} \overline{\mathcal{D}}^{\dot{\gamma}) k} \overline{\mathcal{D}}_{i k}-8 \varepsilon^{\dot{\alpha}(\dot{\beta}} \bar{S}_{i j} \overline{\mathcal{D}}^{\dot{\gamma}) j}-8 \varepsilon^{\dot{\alpha}(\dot{\beta}} \bar{Y}^{\dot{\gamma}) \dot{\delta}} \overline{\mathcal{D}}_{\dot{\delta} i}+6 \bar{Y}^{(\dot{\alpha} \dot{\beta}} \overline{\mathcal{D}}_{i}^{\dot{\gamma})}\right) U, \\
\overline{\mathcal{D}}^{\dot{\alpha} \dot{\beta}} \overline{\mathcal{D}}_{\dot{\alpha} \dot{\beta}} U & =-\left(\overline{\mathcal{D}}^{i j} \overline{\mathcal{D}}_{i j}-8 \bar{S}^{i j} \overline{\mathcal{D}}_{i j}+8 \bar{Y}^{\dot{\gamma} \dot{\delta}} \overline{\mathcal{D}}_{\dot{\gamma} \dot{\delta}}-16\left(\overline{\mathcal{D}}_{\dot{\alpha} i} \bar{S}^{i j}\right) \overline{\mathcal{D}}_{j}^{\dot{\alpha}}\right) U,
\end{aligned}
$$

with $U$ a scalar superfield.

Now comes the crucial point of our analysis. Given an arbitrary covariantly antichiral scalar superfield $\overline{\mathcal{L}}_{\mathrm{c}}$, eq. (3.9), one can check that the four-form $J$ with components (3.8a) -(3.8e is closed 5 At this stage, therefore, we can completely forget about the on-shell vector multiplet $F$ and the explicit realization for $\overline{\mathcal{L}}_{\mathrm{c}}$ in terms of $\bar{W}$ given.

Using the closed four-form $J$ constructed, we generate a locally supersymmetric action in accordance with the general rule (1.6). In complete analogy with the $\mathcal{N}=1$ case, we define the component vierbein $e_{m}{ }^{a}:=\left.E_{m}{ }^{a}\right|_{\theta=0}$ and the gravitino $\Psi_{m_{i}}^{\alpha}:=\left.2 E_{m_{i}}{ }^{\alpha}\right|_{\theta=0}$. Then,

\footnotetext{
${ }^{5}$ In the flat superspace limit, our four-form $J$ reduces to the so-called "chiral" four-form constructed in [31] (this observation is not quite obvious to make, however, because of the ingenious notation adopted in [31]).
} 
the resulting action is

$$
\begin{aligned}
S_{c}=\int & \mathrm{d}^{4} x e\left(\frac{1}{96} \overline{\mathcal{D}}^{i j} \overline{\mathcal{D}}_{i j}-\frac{1}{96} \overline{\mathcal{D}}^{\dot{\alpha} \dot{\beta}} \overline{\mathcal{D}}_{\dot{\alpha} \dot{\beta}}+\frac{2}{3} \bar{S}^{i j} \overline{\mathcal{D}}_{i j}-\frac{1}{3} \bar{Y}^{\dot{\gamma} \dot{\delta}} \overline{\mathcal{D}}_{\dot{\gamma} \dot{\delta}}+\frac{2}{3}\left(\overline{\mathcal{D}}_{\dot{\beta}}^{i} \bar{S}_{i j}\right) \overline{\mathcal{D}}^{\dot{\beta} j}\right. \\
& +\frac{1}{6}\left(\overline{\mathcal{D}}^{i j} \bar{S}_{i j}\right)+3 \bar{S}^{i j} \bar{S}_{i j}-\bar{Y}^{\dot{\alpha} \dot{\beta}} \bar{Y}_{\dot{\alpha} \dot{\beta}}+W^{\alpha \beta} W_{\alpha \beta} \\
& -\frac{\dot{i}}{6} \Psi_{d l}^{\delta}\left(\sigma^{d}\right)_{\delta \dot{\alpha}}\left(\frac{1}{4} \overline{\mathcal{D}}_{q}^{\dot{\alpha}} \overline{\mathcal{D}}^{l q}-\frac{1}{4} \overline{\mathcal{D}}_{\dot{\beta}}^{l} \overline{\mathcal{D}}^{\dot{\beta} \dot{\alpha}}+7 \bar{S}^{l q} \overline{\mathcal{D}}_{q}^{\dot{\alpha}}-5 \bar{Y}^{\dot{\alpha} \dot{\beta}} \overline{\mathcal{D}}_{\dot{\beta}}^{l}+4\left(\overline{\mathcal{D}}_{q}^{\dot{\alpha}} \bar{S}^{l q}\right)\right) \\
& +\frac{\dot{1}}{2} \Psi_{d l}^{\delta}\left(\tilde{\sigma}^{d}\right)^{\dot{\alpha} \alpha} W_{\alpha \delta} \overline{\mathcal{D}}_{\dot{\alpha}}^{l} \\
& +\Psi_{c k}^{\gamma} \Psi_{d l}^{\delta}\left(\frac{1}{4}\left(\sigma^{c d}\right)_{\gamma \delta} \overline{\mathcal{D}}^{k l}+2\left(\sigma^{c d}\right)_{\gamma \delta} \bar{S}^{k l}-\varepsilon_{\gamma \delta} \varepsilon^{k l}\left(\sigma^{c d}\right)_{\alpha \beta} W^{\alpha \beta}\right. \\
& \left.\quad-\frac{1}{8} \varepsilon_{\gamma \delta} \varepsilon^{k l}\left(\tilde{\sigma}^{c d}\right)_{\dot{\beta} \dot{\gamma}} \overline{\mathcal{D}}^{\dot{\beta} \dot{\gamma}}-\varepsilon_{\gamma \delta} \varepsilon^{k l}\left(\tilde{\sigma}^{c d}\right)_{\dot{\alpha} \dot{\beta}} \bar{Y}^{\dot{\alpha} \dot{\beta}}\right) \\
& \left.\frac{1}{4} \varepsilon^{a b c d}\left(\sigma_{a}\right)_{\beta \dot{\alpha}} \Psi_{b j}^{\beta} \Psi_{c k}^{\gamma} \Psi_{d \gamma}^{k} \overline{\mathcal{D}}^{\dot{\alpha} j}+\frac{i}{4} \varepsilon^{a b c d} \Psi_{a i}^{\alpha} \Psi_{b \alpha}^{i} \Psi_{c j}^{\beta} \Psi_{d \beta}^{j}\right)\left.\overline{\mathcal{L}}_{\mathrm{c}}\right|_{\theta=0} .
\end{aligned}
$$

This component action was first computed by Müller [8] using different techniques. Its derivation using the ectoplasm approach is one of the main results of our paper.

The covariantly antichiral scalar superfield $\overline{\mathcal{L}}_{\mathrm{c}}$ can be represented in terms of an unconstrained scalar superfield $\mathcal{L}$ as follows [7, 13]

$$
\begin{aligned}
\overline{\mathcal{L}}_{\mathrm{c}} & =\Delta \mathcal{L}, \\
\Delta & =\frac{1}{96}\left(\left(\mathcal{D}^{i j}+16 S^{i j}\right) \mathcal{D}_{i j}-\left(\mathcal{D}^{\alpha \beta}-16 Y^{\alpha \beta}\right) \mathcal{D}_{\alpha \beta}\right) \\
& =\frac{1}{96}\left(\mathcal{D}_{i j}\left(\mathcal{D}^{i j}+16 S^{i j}\right)-\mathcal{D}_{\alpha \beta}\left(\mathcal{D}^{\alpha \beta}-16 Y^{\alpha \beta}\right)\right),
\end{aligned}
$$

where we have defined

$$
\mathcal{D}_{\alpha \beta}:=\mathcal{D}_{(\alpha}^{k} \mathcal{D}_{\beta) k}, \quad \mathcal{D}_{i j}:=\mathcal{D}_{(i}^{\gamma} \mathcal{D}_{j) \gamma}
$$

In the special case when $\mathcal{L}$ is real, $\overline{\mathcal{L}}=\mathcal{L}$, eq. (3.12) constitutes the component of the general action

$$
\int \mathrm{d}^{4} x \mathrm{~d}^{4} \theta \mathrm{d}^{4} \bar{\theta} E \mathcal{L}, \quad E=\operatorname{Ber}\left(E_{M}{ }^{A}\right)
$$

It can be brought to a manifestly real form by adding to the right-hand side of (3.12) its complex conjugate.

\section{Discussion}

The traditional approaches to the component reduction in four-dimensional $\mathcal{N}=1$ supergravity are reviewed in the textbooks [2, 3, 9]. These approaches are known to be 
extremely laborious. Our derivation of the component action principle (2.13) took only a few hours of calculation, and its technical description requires half a page only. This shows that the ectoplasm method becomes very efficient if the problem of constructing a required closed super $d$-form (in the case of $d$ space-time dimensions) can be re-cast as that of engineering this superform from some closed superforms of lower rank. This idea was successfully applied in section 3 to construct the closed four-form (3.7) $-(3.9)$ in $\mathcal{N}=2$ conformal supergravity, which is associated with an arbitrary covariantly antichiral scalar superfield $\overline{\mathcal{L}}_{\mathrm{c}}$ and generates the locally supersymmetric action (3.12). The four-form (3.7)-(3.9) is a new original result derived for the first time in the present paper. As to the $\mathcal{N}=2$ chiral action (3.12), it was computed twenty years ago by Müller 6 [8] using a technique closely resembling the normal coordinate construction of [13]. Our derivation of the action (3.12) is much more simpler as compared with the calculation in [8].

With the component action (3.12) at our disposal, the projective-superspace formulation for $\mathcal{N}=2$ matter-coupled supergravity given in [13, 26, 27, 32] is completely developed. In particular, any $\mathcal{N}=2$ supergravity-matter action can be readily reduced to components.

\section{Acknowledgements:}

The work of SJG and GT-M is supported by the endowment of the John S. Toll Professorship, the University of Maryland Center for String \& Particle Theory, and National Science Foundation Grant PHY-0354401. GT-M is happy to thank the School of Physics at the University of Western Australia for the kind hospitality and support during part of this work.

\section{A $\quad \mathrm{N}=1$ old minimal supergravity}

Here we collect the key relations used in this paper concerning the superspace geometry within the old minimal formulation for $\mathcal{N}=1$ supergravity, see [9] for more details. Our notation and conventions correspond to [9]; they are similar to those used in [2] except for the normalization of the Lorentz generators, including a sign definition of the sigmamatrices $\sigma_{a b}$ and $\tilde{\sigma}_{a b}$.

The superspace geometry is described by covariant derivatives

$$
\mathcal{D}_{A}=\left(\mathcal{D}_{a}, \mathcal{D}_{\alpha}, \overline{\mathcal{D}}^{\dot{\alpha}}\right)=E_{A}+\Omega_{A}, \quad \Omega_{A}=\frac{1}{2} \Omega_{A}{ }^{b c} M_{b c}=\Omega_{A}{ }^{\beta \gamma} M_{\beta \gamma}+\bar{\Omega}_{A}{ }^{\dot{\beta} \dot{\gamma}} \bar{M}_{\dot{\beta} \dot{\gamma}},
$$

\footnotetext{
${ }^{6}$ It was the last paper on supergravity written by Martin Müller.
} 
with $\Omega_{A}$ the Lorentz connection and $M_{b c} \Leftrightarrow\left(M_{\beta \gamma}, \bar{M}_{\dot{\beta} \dot{\gamma}}\right)$ the Lorentz generators,

$$
\left[M_{\beta \gamma}, \mathcal{D}_{\alpha}\right]=\varepsilon_{\alpha(\beta} \mathcal{D}_{\gamma)}, \quad\left[\bar{M}_{\dot{\beta} \dot{\gamma}}, \mathcal{D}_{\alpha}\right]=0 .
$$

The covariant derivatives obey the following algebra:

$$
\begin{aligned}
\left\{\overline{\mathcal{D}}_{\dot{\alpha}}, \overline{\mathcal{D}}_{\dot{\beta}}\right\}= & 4 R \bar{M}_{\dot{\alpha} \dot{\beta}}, \\
\left\{\mathcal{D}_{\alpha}, \overline{\mathcal{D}}_{\dot{\beta}}\right\}= & -2 \mathrm{i} \mathcal{D}_{\alpha \dot{\beta}}, \\
{\left[\overline{\mathcal{D}}_{\dot{\alpha}}, \mathcal{D}_{\beta \dot{\beta}}\right]=} & -\mathrm{i} \varepsilon_{\dot{\alpha} \dot{\beta}}\left(R \mathcal{D}_{\beta}+G_{\beta}{ }^{\dot{\gamma}} \overline{\mathcal{D}}_{\dot{\gamma}}\right)-\mathrm{i}\left(\mathcal{D}_{\beta} R\right) \bar{M}_{\dot{\alpha} \dot{\beta}} \\
& +\mathrm{i} \varepsilon_{\dot{\alpha} \dot{\beta}}\left(\overline{\mathcal{D}}^{\dot{\gamma}} G_{\beta}{ }^{\dot{\delta}}\right) \bar{M}_{\dot{\gamma} \dot{\delta}}-2 \mathrm{i} \varepsilon_{\dot{\alpha} \dot{\beta}} W_{\beta}^{\gamma \delta} M_{\gamma \delta},
\end{aligned}
$$

where the tensors $R, G_{a}=\bar{G}_{a}$ and $W_{\alpha \beta \gamma}=W_{(\alpha \beta \gamma)}$ satisfy the Bianchi identities

$$
\overline{\mathcal{D}}_{\dot{\alpha}} R=\overline{\mathcal{D}}_{\dot{\alpha}} W_{\alpha \beta \gamma}=0, \quad \overline{\mathcal{D}}^{\dot{\gamma}} G_{\alpha \dot{\gamma}}=\mathcal{D}_{\alpha} R, \quad \mathcal{D}^{\gamma} W_{\alpha \beta \gamma}=\mathrm{i} \mathcal{D}_{(\alpha}{ }^{\dot{\gamma}} G_{\beta) \dot{\gamma}} .
$$

\section{B $\quad \mathrm{N}=2$ conformal supergravity}

This appendix contains a summary of the superspace geometry corresponding to $\mathcal{N}=2$ conformal supergravity, see [26] for more details. Consider a curved $4 \mathrm{D} \mathcal{N}=$ 2 superspace $\mathcal{M}^{4 \mid 8}$ parametrized by local bosonic $(x)$ and fermionic $(\theta, \bar{\theta})$ coordinates $z^{M}=\left(x^{m}, \theta_{\imath}^{\mu}, \bar{\theta}_{\dot{\mu}}^{\imath}\right)$, where $m=0,1, \cdots, 3, \mu=1,2, \dot{\mu}=1,2$ and $\imath=\underline{1}, \underline{2}$. The Grassmann variables $\theta_{\imath}^{\mu}$ and $\bar{\theta}_{\dot{\mu}}^{\imath}$ are related to each other by complex conjugation: $\bar{\theta}_{\imath}^{\mu}=\bar{\theta}^{\dot{\mu} \imath}$. Following [28], the structure group is chosen to be $\mathrm{SL}(2, \mathbb{C}) \times \mathrm{SU}(2)$, and the covariant derivative $\mathcal{D}_{A}=\left(\mathcal{D}_{a}, \mathcal{D}_{\alpha}^{i}, \overline{\mathcal{D}}_{i}^{\dot{\alpha}}\right)$ have the form

$$
\mathcal{D}_{A}=E_{A}+\Omega_{A}{ }^{\beta \gamma} M_{\beta \gamma}+\bar{\Omega}_{A}{ }^{\dot{\beta} \dot{\gamma}} \bar{M}_{\dot{\beta} \dot{\gamma}}+\Phi_{A}^{k l} J_{k l} .
$$

Here $J_{k l}=J_{l k}$ are the generators of $\mathrm{SU}(2)$, and $\Phi_{A}{ }^{k l}(z)$ the corresponding connection. The action of the $\mathrm{SU}(2)$ generators on the covariant derivatives is defined as follows:

$$
\left[J_{k l}, \mathcal{D}_{\alpha}^{i}\right]=-\delta^{i}{ }_{(k} \mathcal{D}_{l) \alpha}, \quad\left[J_{k l}, \overline{\mathcal{D}}_{i}^{\dot{\alpha}}\right]=-\varepsilon_{i(k} \overline{\mathcal{D}}_{l)}^{\dot{\alpha}} .
$$

The covariant derivatives obey the (anti)commutation relations

$$
\begin{aligned}
& \left\{\overline{\mathcal{D}}_{i}^{\dot{\alpha}}, \overline{\mathcal{D}}_{j}^{\dot{\beta}}\right\}=-4 \bar{S}_{i j} \bar{M}^{\dot{\alpha} \dot{\beta}}-2 \varepsilon_{i j} \varepsilon^{\dot{\alpha} \dot{\beta}} \bar{Y}^{\dot{\gamma} \dot{\delta}} \bar{M}_{\dot{\gamma} \dot{\delta}}-2 \varepsilon_{i j} \varepsilon^{\dot{\alpha} \dot{\beta}} W^{\gamma \delta} M_{\gamma \delta} \\
& -2 \varepsilon_{i j} \varepsilon^{\dot{\alpha} \dot{\beta}} \bar{S}^{k l} J_{k l}-4 \bar{Y}^{\dot{\alpha} \dot{\beta}} J_{i j} \\
& \left\{\mathcal{D}_{\alpha}^{i}, \overline{\mathcal{D}}_{j}^{\dot{\beta}}\right\}=-2 \mathrm{i} \delta_{j}^{i}\left(\sigma^{c}\right)_{\alpha}{ }^{\dot{\beta}} \mathcal{D}_{c}+4 \delta_{j}^{i} G^{\delta \dot{\beta}} M_{\alpha \delta}+4 \delta_{j}^{i} G_{\alpha \dot{\gamma}} \bar{M}^{\dot{\gamma} \dot{\beta}}+8 G_{\alpha}{ }^{\dot{\beta}} J^{i}{ }_{j}, \\
& {\left[\mathcal{D}_{a}, \overline{\mathcal{D}}_{j}^{\dot{\beta}}\right]=-\mathrm{i}\left(\sigma_{a}\right)_{\alpha}{ }^{(\dot{\beta}} G^{\alpha \dot{\gamma})} \overline{\mathcal{D}}_{\dot{\gamma} j}+\frac{\mathrm{i}}{2}\left(\left(\tilde{\sigma}_{a}\right)^{\dot{\beta} \gamma} \bar{S}_{j k}-\varepsilon_{j k}\left(\sigma_{a}\right)_{\alpha}{ }^{\dot{\beta}} W^{\alpha \gamma}-\varepsilon_{j k}\left(\sigma_{a}\right)^{\gamma} \bar{Y}^{\dot{\alpha} \dot{\beta}}\right) \mathcal{D}_{\gamma}^{k}} \\
& +\frac{\mathrm{i}}{2}\left(\left(\sigma_{a}\right)_{\delta}{ }^{\dot{\beta}} T_{c d_{j}}{ }^{\delta}+\left(\sigma_{c}\right)_{\delta} \dot{\beta} T_{a d j} \delta-\left(\sigma_{d}\right)_{\delta} \dot{\beta} T_{a c j}^{\delta}\right) M^{c d} \\
& +\frac{\mathrm{i}}{2}\left(-\left(\sigma_{a}\right)^{\gamma} \dot{\gamma} \delta_{j}^{(k} \mathcal{D}_{\gamma}^{l)} \bar{Y}^{\dot{\beta} \dot{\gamma}}-\left(\sigma_{a}\right)_{\gamma}^{\dot{\beta}} \delta_{j}^{(k} \mathcal{D}_{\delta}^{l)} W^{\gamma \delta}+\frac{1}{2}\left(\sigma_{a}\right)_{\alpha} \dot{\beta} \mathcal{D}_{j}^{\alpha} \bar{S}^{k l}\right) J_{k l},
\end{aligned}
$$


where

$$
T_{a b \dot{\gamma}}^{k}=-\frac{1}{4}\left(\sigma_{a b}\right)^{\alpha \beta} \overline{\mathcal{D}}_{\dot{\gamma}}^{k} Y_{\alpha \beta}+\frac{1}{4}\left(\tilde{\sigma}_{a b}\right)^{\dot{\alpha} \dot{\beta}} \overline{\mathcal{D}}_{\dot{\gamma}}^{k} \bar{W}_{\dot{\alpha} \dot{\beta}}-\frac{1}{6}\left(\tilde{\sigma}_{a b}\right)_{\dot{\gamma} \dot{\delta}} \overline{\mathcal{D}}_{l}^{\dot{\delta}} S^{k l}
$$

Here the real four-vector $G_{\alpha \dot{\alpha}}$ and the complex tensors $S^{i j}=S^{j i}, W_{\alpha \beta}=W_{\beta \alpha}, Y_{\alpha \beta}=Y_{\beta \alpha}$ obey the Bianchi identities:

$$
\begin{aligned}
& \overline{\mathcal{D}}_{k}^{\dot{\alpha}} \bar{S}^{k l}+\overline{\mathcal{D}}_{\dot{\gamma}}^{l} \bar{Y}^{\dot{\gamma} \dot{\alpha}}=0, \quad \overline{\mathcal{D}}_{(i}^{\dot{\alpha}} \bar{S}_{j k)}=\mathcal{D}_{(i}^{\alpha} \bar{S}_{j k)}=0, \quad \overline{\mathcal{D}}_{i}^{(\dot{\alpha}} \bar{Y}^{\dot{\beta} \dot{\gamma})}=0, \quad \overline{\mathcal{D}}_{i}^{\dot{\alpha}} W^{\beta \gamma}=0, \\
& \overline{\mathcal{D}}_{i}^{\dot{\alpha}} G^{\gamma \dot{\beta}}=\frac{1}{4} \mathcal{D}_{i}^{\gamma} \bar{Y}^{\dot{\alpha} \dot{\beta}}-\frac{1}{12} \varepsilon^{\dot{\alpha} \dot{\beta}} \mathcal{D}^{\gamma l} \bar{S}_{i l}+\frac{1}{4} \varepsilon^{\dot{\alpha} \dot{\beta}} \mathcal{D}_{\delta i} W^{\gamma \delta}, \\
& \mathcal{D}_{\gamma}^{k} \mathcal{D}_{\delta k} W^{\gamma \delta}-\overline{\mathcal{D}}_{\dot{\gamma} k} \overline{\mathcal{D}}_{\dot{\delta}}^{k} \bar{W}^{\dot{\gamma} \dot{\delta}}=4 W^{\alpha \beta} Y_{\alpha \beta}-4 \bar{W}_{\dot{\alpha} \dot{\beta}} \bar{Y}^{\dot{\alpha} \dot{\beta}}
\end{aligned}
$$

\section{References}

[1] J. Wess and B. Zumino, "The component formalism follows from the superspace formulation of supergravity," Phys. Lett. B 79, 394 (1978).

[2] J. Wess and J. Bagger, Supersymmetry and Supergravity, Princeton Univ. Press, 1992.

[3] S. J. Gates Jr., M. T. Grisaru, M. Roček and W. Siegel, Superspace, or One Thousand and One Lessons in Supersymmetry, Benjamin/Cummings (Reading, MA), 1983, hep-th/0108200.

[4] U. Lindström, A. Karlhede and M. Roček, "The component gauges in supergravity," Nucl. Phys. B 191, 549 (1981).

[5] M. Müller, "The density multiplet in superspace," Z. Phys. C 16, 41 (1982).

[6] C. Ramirez, "The nonlinear realizations of the supersymmetry in the unconstrained curved superspace," Z. Phys. C 28, 281 (1985); "The structure of the new superspace," Z. Phys. C 33, 455 (1987); "The realizations of local supersymmetry," Annals Phys. 186, 43 (1988).

[7] M. Müller, Consistent Classical Supergravity Theories, (Lecture Notes in Physics, Vol. 336), Springer, Berlin, 1989.

[8] M. Müller, "Off-shell supergravity actions," Preprint MPI-PAE/PTh 64/89, Munich, 1989.

[9] I. L. Buchbinder and S. M. Kuzenko, Ideas and Methods of Supersymmetry and Supergravity, Or a Walk Through Superspace, IOP, Bristol, 1998.

[10] J. J. Atick and A. Dhar, Theta-expansion and light-cone gauge fixing in curved superspace sigma-models," Nucl. Phys. B 284, 131 (1987).

[11] I. N. McArthur, "Superspace normal coordinates," Class. Quant. Grav. 1, 233 (1984).

[12] M. T. Grisaru, M. E. Knutt-Wehlau and W. Siegel, "A superspace normal coordinate derivation of the density formula," Nucl. Phys. B 523, 663 (1998) hep-th/9711120.

[13] S. M. Kuzenko and G. Tartaglino-Mazzucchelli, "Different representations for the action principle in 4D N = 2 supergravity," JHEP 0904, 007 (2009) [arXiv:0812.3464 [hep-th]].

[14] D. Tsimpis, "Curved 11D supergeometry," JHEP 0411, 087 (2004) hep-th/0407244. 
[15] S. J. Gates Jr., "Ectoplasm has no topology: The prelude," in Supersymmetries and Quantum Symmetries, J. Wess and E. A. Ivanov (Eds.), Springer, Berlin, 1999, p. 46, arXiv:hep-th/9709104 "Ectoplasm has no topology," Nucl. Phys. B 541, 615 (1999) arXiv:hep-th/9809056.

[16] S. J. Gates Jr., M. T. Grisaru, M. E. Knutt-Wehlau and W. Siegel, "Component actions from curved superspace: Normal coordinates and ectoplasm," Phys. Lett. B 421, 203 (1998) hep-th/9711151].

[17] A. V. Gaiduk, O. M. Khudaverdian and A. S. Schwarz, "Integration over surfaces in superspace," Theor. Math. Phys. 52, 862 (1982) [Teor. Mat. Fiz. 52, 375 (1982)].

[18] M. A. Baranov and A. S. Schwarz, "Characteristic classes of supergauge fields," Functional Anal. Appl. 18, 130 (1984) [Funkts. Anal. Prilozh., 18, 53 (1984)]; "Cohomology of supermanifolds," Functional Anal. Appl. 18, 236 (1984) [Funkts. Anal. Prilozh., 18, 69 (1985)].

[19] T. Voronov, "Geometric integration theory on supermanifolds," Sov. Sci. Rev. C: Math. Phys. 9, 1 (1992).

[20] O. M. Khudaverdian and A. P. Nersessian, "Batalin-Vilkovisky formalism and integration theory on manifolds," J. Math. Phys. 37, 3713 (1996) arXiv:hep-th/9507026.

[21] O. M. Khudaverdian, "Batalin-Vilkovisky formalism and odd symplectic geometry," in Geometry and Integrable Models, P. N. Pyatov and S. N. Solodukhin (Eds.), World Scientific, Singapore, 1996, p. 144, arXiv:hep-th/9508174.

[22] S. J. Gates Jr. and G. Tartaglino-Mazzucchelli, "Ectoplasm \& superspace integration measure for 2D supergravity with four spinorial supercurrents," arXiv:0907.5264 [hep-th].

[23] N. Berkovits and P. S. Howe, "The cohomology of superspace, pure spinors and invariant integrals," arXiv:0803.3024 [hep-th].

[24] G. Bossard, P. S. Howe and K. S. Stelle, "The ultra-violet question in maximally supersymmetric field theories," Gen. Rel. Grav. 41, 919 (2009) [arXiv:0901.4661 [hep-th]]; "A note on the UV behaviour of maximally supersymmetric Yang-Mills theories," arXiv:0908.3883 [hep-th].

[25] W. Siegel, Fields, arXiv:hep-th/9912205.

[26] S. M. Kuzenko, U. Lindström, M. Roček and G. Tartaglino-Mazzucchelli, "4D N=2 supergravity and projective superspace," JHEP 0809, 051 (2008) arXiv:0805.4683.

[27] S. M. Kuzenko, U. Lindström, M. Roček and G. Tartaglino-Mazzucchelli, "On conformal supergravity and projective superspace," JHEP 0908, 023 (2009) arXiv:0905.0063 [hepth]].

[28] R. Grimm, "Solution of the Bianchi identities in SU(2) extended superspace with constraints," in Unification of the Fundamental Particle Interactions, S. Ferrara, J. Ellis and P. van Nieuwenhuizen (Eds.), Plenum Press, New York, 1980, pp. 509-523.

[29] P. S. Howe, "Supergravity in superspace," Nucl. Phys. B 199, 309 (1982). 
[30] R. Grimm, M. Sohnius and J. Wess, "Extended supersymmetry and gauge theories," Nucl. Phys. B 133, 275 (1978).

[31] T. Biswas and W. Siegel, "N=2 harmonic superforms, multiplets and actions," JHEP 0111, 004 (2001) arXiv:hep-th/0105084.

[32] S. M. Kuzenko, "On N = 2 supergravity and projective superspace: Dual formulations," Nucl. Phys. B 810, 135 (2009) arXiv:0807.3381 [hep-th]]. 